\title{
ZUR GRUNDLEGUNG DER PHYTOMEDIZIN
}

Gekürzte Fassung eines am 3. V. 1962 vor Vertretern der Landwirtschaftlichen Fakultät der Universität Helsinki gehaltenen Vortrages.

\section{E. MüHLE}

\section{Institut für Phytopathologie der Karl-Marx-Universität Leipzig}

Der Wissenschaftszweig, auf den ich heute Ihre Aufmerksamkeit lenken möchte, ist dadurch gekennzeichnet, dass er gegenwärtig noch kein festes Gefüge darstellt, sondern uns als ein Gebilde begegnet, das sich aus mehreren nebeneinander gewachsenen Säulen zusammensetzt. Die beiden wichtigsten dieser Säulen sind die Phytopathologie und die angewandte Entomologie. Wenn wir aber die Tätigkeit der hier wirksamen beiden Gruppen von Wissenschaftlern kritisch beleuchten, so müssen wir feststellen, dass für viele bisher eigentlich nicht die Pflanze im Mittelpunkt ihres wissenschaftlichen Bemühens gestanden, sondern dass sie viel entscheidender die jeweilige Schadursache bzw. der Schaderreger interessiert hat. Geschichtlich gesehen entwickelten sich dabei ausser der Phytopathologie im engeren Sinne und der Phyto-Entomologie allmählich noch eine Phyto-Virologie, eine PhytoNematologie, eine Phyto-Acarologie und einige weitere kleinere Spezialgebiete.

Ohne das hier offenbar gewordene Problem gelöst zu haben, prägte man in Deutschland für diejenigen, die sich mit der kranken Pflanze und ihren Schaderregern befassten, die Berufsbezeichnung "Pflanzenarzt». Damit hat man zwar äusserlich eine Parallele zu denen hergestellt, die es ebenfalls mit kranken oder geschädigten Lebewesen zu tun haben, mit dem Humanmediziner und dem Veterinärmediziner, aber der eigentliche reale Hintergrund, vor dem das hätte geschehen können, nämlich die einheitliche Wissenschaft, die das gestattet hätte, war noch nicht begründet.

Wen wir alle, die sich um die durch Krankheiten und Schädlinge in Mitleidenschaft gezogene Pflanze bemühen, als Pflanzenärzte, als Phytomediziner bezeichnen, gibt sich ein vorwärtsweisender Anknüpfungspunkt in der Tatsache, dass man das Betätigungsobjekt eines jeden Arztes schlechthin als "Patient» bezeichnet. Es gibt einen menschlichen Patienten, und wir sprechen auch von einem tierischen Patienten. Was liegt näher, in unserem Falle nun auch von einem pflanzlichen Patienten zu 
sprechen? Und warum folgert man dann nicht weiter: Für den Arzt des menschlichen Patienten ist die Humanmedizin die massgebliche und alle Einzeldisziplinen zusammenfassende Wissenschaft, für den Arzt des tierischen Patienten ist es die Veterinärmedizin; warum fordert man nicht, dass bei der für den Arzt des pflanzlichen Patienten massgeblichen und die Einzeldisziplinen zusammenfassenden Wissenschaft allgemein von einer "Phytomedizin» gesprochen wird?

Wenn man sich das Krankheitsgepräge bei Mensch, Tier und Pflanze vergegenwärtigt, so begegnen dem Humanmediziner an seinem Patienten zunächst in besonders grossem Umfange physiologische Krankheiten, ihm begegnen ferner viröse und parasitär bedingte Funktionsstörungen und morphologische Veränderungen der inneren Organe, ihm begegnen aber auch äussere Verletzungen und ihm begegnen schliesslich Erscheinungen, die durch tierische Parasiten hervorgerufen werden. Sehr ähnlich ist dies dem, was dem Tierarzt bei seinem Patienten begegnet. Dabei ist zu erkennen, dass die parasitologische Schädigungsgruppe in der Veterinärmedizin bereits einen grösseren Raum einnimmt als in der Humanmedizin.

Und wenn wir uns die Pflanze als Patient anschauen? Auch bei ihr gibt es physiologische Krankheiten, krankhafte Funktionsstörungen und morphologische Veränderungen. Einen viel grösseren Raum als bei Mensch und Tier nehmen bei der Pflanze dann bereits die Virosen, Bakteriosen und Mykosen ein. Und noch grösser als beim Tier wird bei der Pflanze die Zahl der tierischen Parasiten. Zu diesen allen kommt schliesslich entsprechend der Stellung der Pflanze in der Natur das fast unübersehbare Heer der Phytophagen, das vor allem deshalb so gross ist, weil die Pflanze als solche primär einem grossen Teil des Tierreiches und dem Menschen zur Nahrung dient.

Aber auch in anderer Beziehung treten hinsichtlich der ärztlichen Betätigungsobjekte bei den einzelnen medizinischen Wissenschaften Unterschiede hervor. Die Humanmedizin hat es mit einer einzigen biologischen Art zu tun, die Tiermedizin hat sich bereits mit einer grossen Zahl von Arten zu befassen und noch grösser ist die Artenzahl, um die sich die Phytomedizin zu kümmern hat. Innerhalb der der Tiermedizin begegnenden Arten sind weiter grosse Unterschiede vorhanden, die sich auf die Möglichkeit beziehen, dem Patienten überhaupt zu helfen. Hier ergeben sich Einschränkungen in der Möglichkeit ärztlicher Betätigung, die bereits vom Menschen zum Tier hin umfassender werden. Ein menschlicher Patient bietet naturgemäss zunächst oft mehr Aussichten für ein erfolgreiches Heilverfahren als ein Tier. Ein Pferd oder eine Kuh bieten weiter andere Aussichten für bestimmte Heilverfahren als ein Kaninchen oder ein Huhn. Noch viel geringer werden die Möglichkeiten, dem Patienten zu helfen, wenn wir schliesslich die Pflanze ins Auge fassen. Aber auch innerhalb des Pflanzenreiches zeigen sich Abstufungen. Einem langjährigen Obstbaum wird im Falle einer Krankheit ganz andere Hilfe zuteil werden können als einer kurzlebigen Senfpflanze.

Die kurze Lebensdauer vieler Nutzpflanzen zwingt uns weiter, die ärztliche Hilfe bei der Pflanze viel umfassender auf vorbeugende Massnahmen, auf den "Schutz" der Pflanzen zu konzentrieren, als das bei Mensch und Tier notwendig ist. Schliesslich muss aber noch eine ganz andere, die ärztliche Betätigung bestimmende 


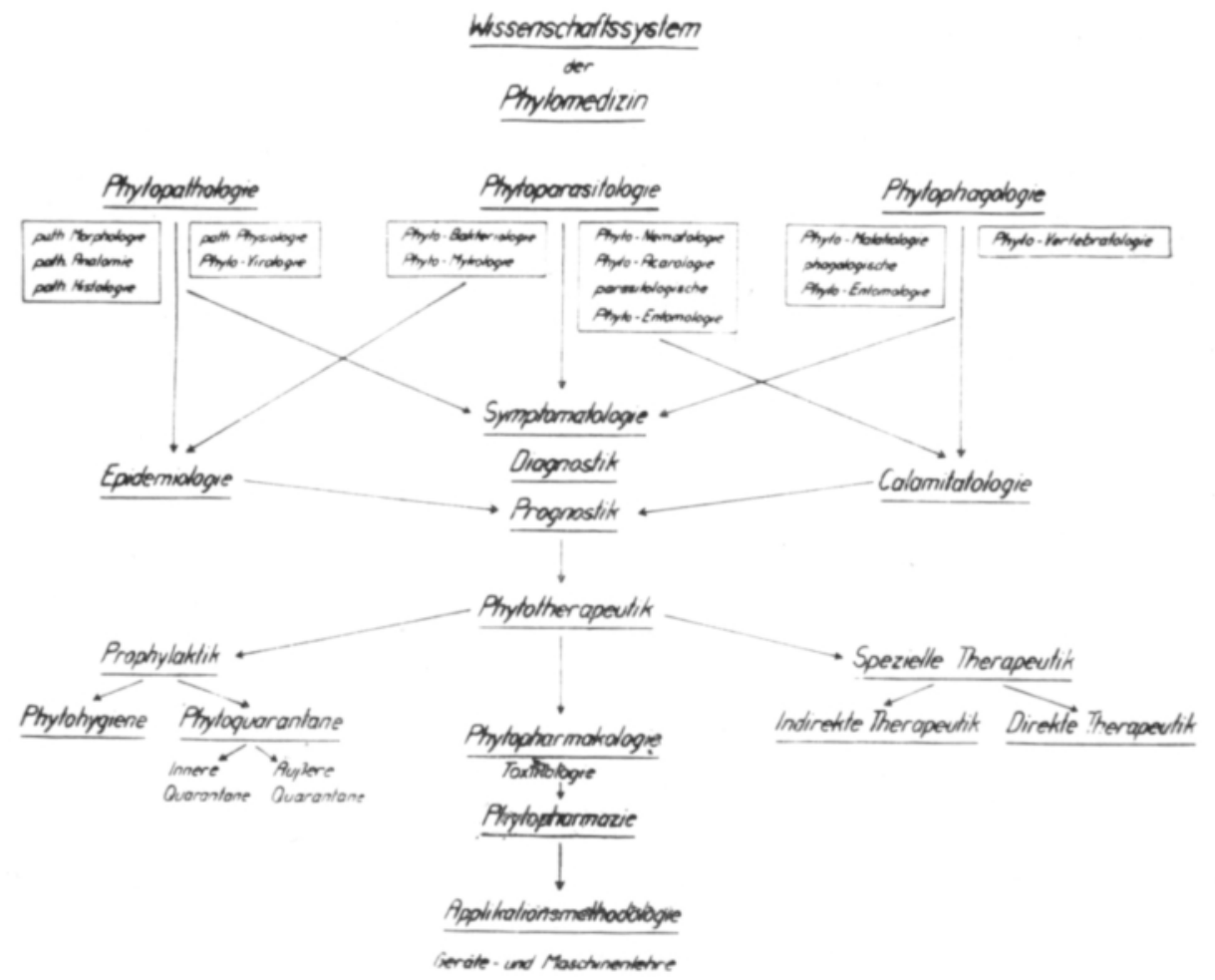

Seite angesprochen werden, ich meine die ökonomische. Der Medizin als der Wissenschaft vom "Heilen» sollte es zwar zunächst um das jeweilige Lebewesen schlechthin gehen, der Humanmedizin um den Menschen, der Veterinärmedizin um das Tier und einer künftigen Phytomedizin sollte es zunächst um die Pflanze schlechthin zu tun sein. Aber wenn wir uns die praktische Seite der jeweiligen Medizin ansehen, so erfährt sie bereits vom Menschen zum Tier eine wesentliche, ökonomisch bestimmte Einengung und diese ökonomische Seite wird dann bei der Pflanze fast vollständig zum bestimmenden Faktor der ausübenden medizinischen Tätigkeit.

Was ist nun gewonnen, wenn wir auf die Einrichtung einer Phytomedizin hinarbeiten? Die sich daraus ergebenden Folgen sind im einzelnen vielleicht noch nicht ganz abzusehen. Sie beginnen sich aber bereits abzuzeichnen, wenn wir daraus dann die ersten Konsequenzen für unsere weitere Betätigung, und zwar sowohl für Forschung und Lehre als auch für die Praxis, ziehen. Im grossen ergibt sich aus unseren Ausführungen zunächst, dass damit eine Wissenschaft vorhanden ist, die die Pflanze allgemein und zwar zunächst ohne den Hintergrund des rechnenden Ökonomen in gleicher Weise zum Objekt eines einheitlichen Bemühens erhebt, wie es die Humanmedizin in bezug auf den Menschen und die Veterinärmedizin in bezug auf das Tier bereits lange getan haben. Phytopathologie im engeren Sinne und noch mehr die sogenannte "Angewandte Entomologie" verlieren dadurch das lose Nebeneinander, das ihnen bisher eigen war und werden mit anderen der anfangs genannten, in gewisser Isolation stehenden Arbeitsgebieten zu Teildisziplinen der sie umfassenden 
Phytomedizin. Das zentrale Objekt ist für sie alle in Zukunft nicht mehr der an der Pflanze angetroffene Krankheitserreger oder Schädling, sondern der von einem krankmachenden oder schädigenden Agens in Mitleidenschaft gezogene pflanzliche Patient.

Unter diesem Gesichtswinkel lässt sich die Phytomedizin vielleicht zunächst in drei grosse Hauptdisziplinen gliedern:

Die erste dieser Grundwissenschaften der Phytomedizin ist die Phytopathologie, und zwar die Phytopathologie im engeren Sinne des Wortes. Für sie ergibt sich, dass vieles, was uns an dem Patienten "Pflanze" zu begegnen vermag, zunächst in der Pflanze selbst begründet sein kann, in Unzulänglichkeiten ihrer Morphologie, Anatomie, Histologie und Physiologie, ausgelöst durch Faktoren ihres Erbgefüges und der unbelebten Umwelt sowie durch das komplizierte Geschehen im Pflanzeninneren. Damit erfährt die Phytopathologie nunmehr inhaltlich eine klare und gegenüber manchen derzeitigen Gepflogenheiten einschränkende Grenzziehung. Sie würde einerseits die pathologische Morphologie, die pathologische Anatomie, die pathologische Histologie und die pathologische Physiologie der Pflanze umfassen. Zur Phytopathologie dürfte darüber hinaus aber auch alles gehören, was an virösen Erscheinungen an und in der Pflanze beobachtet werden kann, d.h. auch die pflanzliche Virologie müsste bei der Phytopathologie im engeren Sinne verbleiben.

Pathologische Erscheinungen im weiteren Sinne werden an der Pflanze dann dadurch hervorgerufen, dass bestimmte pflanzliche und tierische Organismengruppen die normale Entwicklung und die Lebensfunktionen der Pflanze beeinträchtigen. Die meisten speziell hier gemeinten Organismen, von denen einige für die Pflanzen durchaus als "Pathogene" eine Rolle spielen, werden allgemein unter dem Begriff "Parasiten» zusammengefasst. Ihrer grossen Zahl und der Einheitlichkeit nach, die in ihrem primären Angriff auf die Pflanze zum Ausdruck kommt, erscheint es ratsam, alles, was durch sie an und in der Pflanze geschieht, von der Pathologie im engeren Sinne abzutrennen und dafür eine zweite grundlegende Teilwissenschaft der Phytomedizin zu bemühen, die Phytoparasitologie.

Von der Phytoparasitologie würden zunächst zwei bisher ausschliesslich von der Phytopathologie bearbeitete pflanzliche Organismengruppen zu übernehmen sein, die phytoparasitären Bakterien und die phytoparasitären Pilze. Damit würden wir zu einer speziellen Phyto-Bakteriologie und zu einer Phyto-Mykologie kommen. Der tierische Sektor der Parasitologie würde die bisher zumeist von der Phytopathologie im alten Sinne bearbeitete Phyto-Nematologie, ferner die Phyto-Acarologie und die parasitogene Phyto-Entomologie umfassen, wobei letztere ausschliesslich die speziell als Parasiten im engeren auftretenden Insekten, d.h. insbesondere alle Pflanzensaftsauger, zu berücksichtigen hätte.

Der tierische Sektor der Phytoparasitologie leitet dann zu anderen, die Pflanze in Mitleidenschaft ziehenden tierischen Organismen über. Diese sind vor allem dadurch gekennzeichnet, dass sie der Pflanze durch ihre Frasstätigkeit Wunden, Verletzungen und Gewebeverluste beibringen. Alles, was diesen Bereich der an dem Patienten "Pflanze» auftretenden Erscheinungen betrifft, würde einer dritten grund- 
legenden Teilwissenschaft der Phytomedizin, der Phytophagologie, zuzuweisen sein. Diese würde sich dann in eine Phyto-Malakologie, eine ausschliesslich die fressenden Insekten berücksichtigende phagogene Phyto-Entomologie und eine Phyto-Vertebratologie aufgliedern lassen.

Aus Phytopathologie und Phytoparasitologie einerseits und aus Phytoparasitologie und Phytophagologie andererseits ergeben sich weiter Wissens- und Forschungsgebiete der Phytomedizin, die vor allem die Massenschädigungen der Pflanzen durch Fremdorganismen zum Gegenstand haben. Soweit dabei die Viren und die genannten pflanzlichen Organismen im Spiel sind, würden wir zu einer phytomedizinischen Epidemiologie kommen, soweit es sich um tierische Organismen handelt, müssten wir an eine phytomedizinische Calamitatologie denken.

Ein weiterer wesentlicher Teil wissenschaftlicher Betätigung würde dem Wissenschaftszweig der Phytomedizin zufallen, der sich mit den beim Patienten "Pflanze" möglichen Behandlungs- und Heilverfahren zu befassen hat. Es ist die Therapeutik im weitesten Sinne. Hierbei würde zwischen dem Teil der Therapeutik zu unterscheiden sein, der die vorbeugenden, d.h. prophylaktischen Verfahren bearbeitet und dem Teil, der einerseits die indirekten, andererseits die direkten therapeutischen Verfahren zu berücksichtigen hat. $\mathrm{Zu}$ den Verfahren der prophylaktischen Therapeutik würde vor allem das grosse Wissenschaftsgebiet der Pflanzenhygiene zu rechnen sein. Zur indirekten Therapeutik müsste man einmal die biologischen Massnahmen im weitesten Sinne zählen, also sowohl die Anwendung der Antibiotika und der antibiotischen Wirkungskomplexe als auch die Massnahmen der biologischen Schädlingsbekämpfung. Zum anderen würden hier wesentliche Teile aus dem Gebiet der unmittelbaren physikalischen und chemischen Bekämpfungsmassnahmen berücksichtigt werden müssen.

Für eine direkte Therapeutik, d.h. für die Möglichkeiten einer wirklichen Heilbehandlung im engeren Sinne, wie sie bei Mensch und Tier eine massgebliche Rolle spielen, lässt die Pflanze auch heute noch wenig Raum. Da sich hier aber, wie jüngere Forschungsbemühungen erkennen lassen, in Zukunft einiges ändern dürfte, muss auch dieses Gebiet der Therapeutik in der Wissenschaftsstruktur einer Phytomedizin bereits voll in Ansatz gebracht werden.

Ergänzend zur Therapeutik würden im Wissenschaftssystem einer Phytomedizin weiter noch eine Phytopharmakologie mit einer Toxikologie sowie eine Phytopharmazie einen wichtigen Platz einzunehmen haben. Anschliessend würde dann die Applikationsmethodologie mit der hier einzubauenden Geräte- und Maschinenlehre zu berücksichtigen sein. Schliesslich müssten auch die Fragen der phytomedizinischen Ökonomie eine gewisse Berücksichtigung erfahren.

Weitere Konsequenzen, die sich aus der Begründung einer Phytomedizin ergeben, dürften dann vor allem die sein, dass auch bald daran gegangen werden müsste, wirkliche "Phytomediziner» zu entwickeln. Dazu genügt es in Zukunft nicht mehr, im Rahmen eines Studiums der Landwirtschaftslehre, der Gartenbauwissenschaft, der Forstwissenschaft oder der Biologie einen Drall in Richtung auf die bisherige "Phytopathologie» zu vermitteln. Bei dem heutigen Umfang unseres Arbeitsgebietes und bei der Fülle der zu bewältigenden Einzelerkenntnisse genügt 
es auch nicht mehr, sich das erforderliche Wissen z.B. durch ein Zusatzstudium anschliessend an ein allgemeines Biologie- oder Landwirtschaftsstudium anzueignen. Dem wirklichen künftigen Phytomediziner muss vielmehr genau so ein selbständiges Vollstudium erschlossen werden, wie es für den Human- oder Veterinärmediziner vorhanden ist.

Der künftig auszubildende Pflanzenarzt muss an seinen Patienten während des Studiums durch alle Wissenschaftszweige herangeführt werden, die sich mit der Pflanze befassen. Die Grundwissenschaft ist für ihn zunächst die Wissenschaft von der Pflanze, die Botanik, mit allen ihren Teildisziplinen. Von den grundlegenden Begleitwissenschaften ist insbesondere die Chemie hervorzuheben. Weitere Grundlagen vermitteln dann die Disziplinen, die die Pflanze vor allem als Kulturobjekt in den Mittelpunkt von Lehre und Forschung stellen, wobei insbesondere dem Ackerbau, dem Pflanzenbau, der Pflanzenernährung, der Pflanzenzüchtung grundsätzliche Bedeutung zukommt.

Erst wenn der Studierende das normale Aussehen der Pflanze, ihre normale Entwicklung und ihr normales Verhalten unter natürlichen oder bestimmten ihr von uns Menschen vorgegebenen Bedingungen und das normale Funktionieren ihrer Organe mit aller Deutlichkeit erkannt hat, kann er sich mit dem Abnormen, dem Pathologischen an ihr befassen.

Wenn sich der Studierende in all diesen Dingen das wissenschaftliche Rüstzeug erworben hat, kann er auch an die Frage herangehen, wie der Pflanze in ihren Lebensnöten zu helfen ist. Er kann sich nun die Mittel und Möglichkeiten zur Bekämpfung der Krankheiten und Schädigungen aneignen, er kann nunmehr dem Patienten Pflanze als Arzt gegenübertreten.

Nur derjenige, der ein spezielles Studium der Phytomedizin nachzuweisen und durch ein Staatsexamen abgeschlossen haben wird, kann in Zukunft voll für sich das Recht in Anspruch nehmen, wirklich "Phytomediziner» oder "Pflanzenarzt" zu sein. Er wird als solcher damit in der Öffentlichkeit die Anerkennung erlangen, die sich sowohl der bisherige "Phytopathologen als auch der sog. "Angewandte Entomologen vielleicht auch in Zukunft niemals ganz verschaffen wird. Und er wird darüber hinaus aber wahrscheinlich auch viel umfassendere Möglichkeiten sehen, dem Patienten "Pflanze» zu helfen und zwar in einer Weise, wie es weder dem bisherigen "Phytopathologen" noch dem bisherigen "Angewandten Entomologen* möglich war. 